\title{
Rock burst hazard assessment of apatite mines on Kola Peninsula by means of local acoustic emission method.
}

\author{
Alexander Rozanov ${ }^{1 *}$, and Andrei Tereshkin ${ }^{2}$ \\ ${ }^{1}$ Saint-Petersburg Mining University, Research Center of Geomechanics and Issues of Mining \\ Industry, Saint-Petersburg, Russia \\ ${ }^{2}$ Mining Institute of Far Easten Branch of Russian Academy of Sciences, Khabarovsk, Russia
}

\begin{abstract}
Various acoustic emission (AE) parameters are used to characterize fracture process in rocks. The conventional statistical values are, for example, AE activity, $b$-factor, and distribution of energy. In order to map AE events the location analysis is used. There are also of interest in seismic analysis such kinematic parameters as corner frequency, seismic moment. To describe spatial pattern of hypocenter distribution a so called correlation integral is applied. In this work we apply spectral-correlation analysis to quantify each $\mathrm{AE}$ waveform with energy-correlation values. To study the behavior of spectral parameters during fracturing we carried out a series of $\mathrm{AE}$ measurements in the areas of extreme mining pressure in the PhosAgro apatite mines. For data acquisition purpose we use a device «Prognoz-L» which has been developed in the Mining Institute of Khabarovsk, Russia, for local stress monitoring.
\end{abstract}

\section{Introduction}

Determination of dynamic mechanical properties of rock materials is very important for evaluation of stability of rock structures under dynamic loads. For example, at different scales (seismic scale, oil or gas reservoirs, mine pillars) rock material is subjected to compressive loads at different strain rates. In all cases rock loses its stability not instantaneously, but gradually approaching to its fault. Hence it becomes very important to predict this instability in rock media which starts to develop from micro-fracturing. This problem leads us to the process zone characterization when making observations of fracture process in rocks. For this purpose the AE method is useful.

In terms of microscale faulting fracture is caused by sudden releas of accumulated strain energy when the local strength of material is exceeded. Natural acoustic waves are radiated by grain scale fracturing events. These waves contain information about fractures. AE studies in controlled laboratory conditions provide data of various AE waveforms $[1,2]$.

In view of importance of rock burst monitoring in mines new technologies of geoacoustic systems are widely introduced nowadays. For example, a multifunctional

* Corresponding author: aleksander.rozanov@mail.ru 
device of mine local control (Prognoz-L) has been developed in the Mining Institute of Khabarovsk [3]. Now it is successfully used in different mines of Russia, one of them is the appatite mine on Kola Peninsula. The calculations of $b$-value, energy and activity of AE are implemented into the device in online mode. One can carry out the post processing of digitized $\mathrm{AE}$ waveforms. In this work the $\mathrm{AE}$ waveforms has been subjected to spectral correlation analysis. With the use of spectral correlation algorithm developed on the basis of numerous laboratory data $[4,5]$ the sequence of stick-slip events [6] has been analised in the work [7]. We distinguished three main patterns of AE spectra: narrowband with high correlation $(\approx 1)$, wideband with low correlation $(<0.5)$, and $\Pi$-pattern of high correlation $(\approx 1)$. The AE data acquired under mining conditions have clearly revealed the transition point from stable process of fracturing to unstable process by AE median frequency and standard deviation observations [8].

The aim of this work is to capture an onset of transition process from stable fracturing to unstable fracturing with the use of cross-correlation analysis. The AE data acquired with the device «Prognoz-L» during mining local pressure control in appatite mine have been used.

\section{Spectral-correlation method}

Historically, Fourier spectral analysis has provided a general method for examining the energy-frequency distribution for a certain time series. As a result, the term "spectrum" has become almost synonymous with the Fourier transform of the data. Although the Fourier transform is valid under the general conditions, there are some important restrictions of the Fourier spectral analysis: the system must be linear; and the data must be strictly periodic or stationary; otherwise, the resulting spectrum will make little physical sense. The far-field seismic phenomena can be approximated by linear systems and so be represented by linear superposition of sinusoid functions adequately. The algorithms of Fourier transform of the signals of finite duration is well developed and the interpretation is quite explicit $[9,10]$.

The most basic method of data analysis is a limited time window-width Fourier spectral analysis. By successively sliding the window along the time axis, one can get a timefrequency distribution. AE signals are converted from time $t$ to frequency $f$ domain using a Finite Fourier Transform. Let us consider two signals $x(t)$ and $y(t)$ and divide each of them into $n_{d}$ equal segments $x_{k}(t)$ and $y_{k}(t)$ with $T$ duration (time windowing). Then the spectral density estimations $G_{X X}(f)$ and $G_{Y Y}(f)$ are produced as follows:

$$
\begin{aligned}
& G_{X X}(f)=\frac{2}{n_{d} T} \sum_{k=1}^{n_{d}}\left|X_{k}(f, T)\right|^{2} \\
& G_{Y Y}(f)=\frac{2}{n_{d} T} \sum_{k=1}^{n_{d}}\left|Y_{k}(f, T)\right|^{2}
\end{aligned}
$$

where $X_{k}(f, T)$ and $Y_{k}(f, T)$ are the Finite Fourier Transforms of $x_{k}(t)$ and $y_{k}(t)$ respectively: 


$$
\begin{aligned}
& X_{k}(f, T)=\int_{0}^{T} x_{k}(t) e^{-i 2 \pi f t} d t \\
& Y_{k}(f, T)=\int_{0}^{T} y_{k}(t) e^{-i 2 \pi f t} d t
\end{aligned}
$$

The cross-spectral density $G_{X Y}(f)$ is a complex function of real variable:

$$
G_{X Y}(f)=\frac{2}{n_{d} T} \sum_{k=1}^{n_{d}} X_{k}^{*}(f, T) Y_{k}(f, T)
$$

where $X_{k}^{*}(f, T)$ is the complex conjugate of $X_{k}(f, T)$.

Using the cross-spectral estimation one can investigate the frequency dependent correlation between two signals of one radiation event or between two sequent signals of two different radiation events. The so-called coherence function allows us to do that. The coherence function is expressed as a ratio of cross-spectral and spectral densities of two relevant signals:

$$
\gamma_{X Y}^{2}(f)=\frac{\left|G_{X Y}(f)\right|^{2}}{G_{X X}(f) G_{Y Y}(f)}
$$

Coherence function controls the similarity of two AE signals - the amplitude of the second signal can change relatively to the first signal, but the dominant frequency and the wave shape must remain the same. In this case a value of coherence function tends to 1 , and in case the similarity is lost it tends to 0 .

The idea of this work is to perform one sequence for all $\mathrm{AE}$ signals registered in a certain day of measurements, and then to divide this sequence into the pairs of sequent $\mathrm{AE}$ signals. The second step is to apply for each $\mathrm{AE}$ pair the cross-correlation analysis according to the equation (6). Finally we obtain the trend of coherence values and compare it with the trend of energy. Coherence value for each $\mathrm{AE}$ pair we associate with the dominant frequency of cross-spectral density. Energy estimation is produced from the spectrum of each $\mathrm{AE}$ signal according to the equation:

$$
E E=\sqrt{\int_{f_{-}}^{f_{+}} G_{X X}(f) d f}
$$

where $f_{-}$and $f_{+}$are the lower and upper limits of a valid signal frequency band. 


\section{Application of spectral-correlation method to AE field data}

On the basis of numerous observations of mine $\mathrm{AE}$ data we suppose that violation of $\mathrm{AE}$ similarity associated with high $\mathrm{AE}$ energy is a reliable warning of transition to unstable phase of fracturing which in turn can lead to rock damage. This supposition we test in the mine area which was presumably under high stress conditions. The controlled mine opening was being developed and at the same time excavated area approached too close in the north. On the other hand a substantial geological inhomogeneity of dyke type occurs about $60 \mathrm{~m}$ to the south. Figure 1 performs a photo of the face of mine opening where AE monitoring has been carried out. AE transducer has been mounted exactly on the wall close to the face of mining.

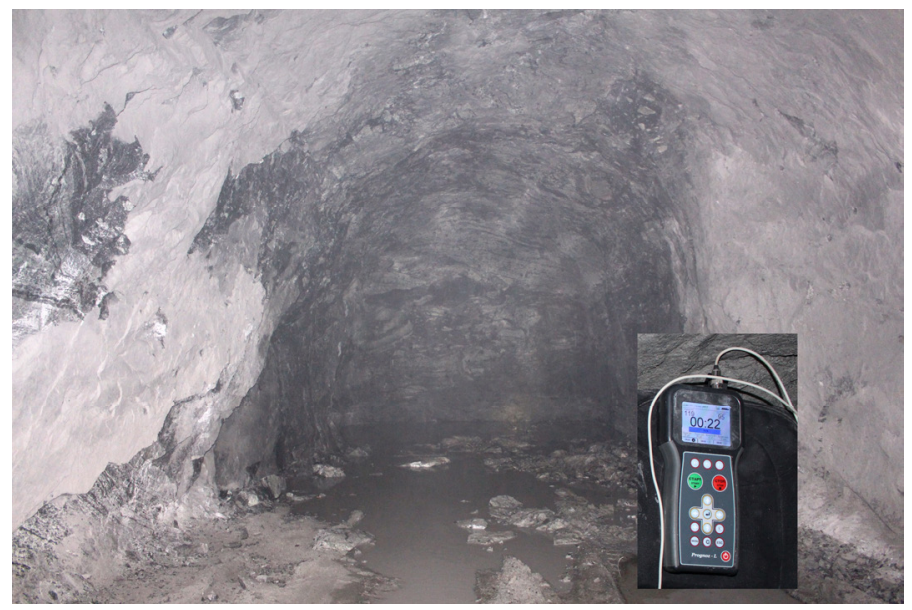

Fig.1. The face of controlled mine opening and the device «Prognoz-L» installed on the site of AE monitoring.

AE monitoring has been performed over the course of three days. The typical mismatch of $\mathrm{AE}$ waveforms (Figure 2b) compared to their correspondence (Figure 2a) is shown in Figure 2. Figure 3 illustrates the cross-correlation calculation of AE waveform similarity respectively. The big circles depict high values of spectral density associated with the dominant frequency of cross-spectrum. One can see that in case of perfect correlation of two AE waveforms the coherence function for big circles approaches 1 (Figure 3a), and when similarity is lost they drop to near zero values of the coherence function (Figure $3 b$ ). This is an example of how to detect the similarity violation with the use of cross-correlation analysis.

Then we combine the data of three days of measurements into one sequence and launch continuous cross-correlation and energy analysis. What we obtain is the $3 \mathrm{D}$ display in time domain of $\mathrm{AE}$ monitoring which detects energy-correlation anomalies evolving in the course of measurements (Figure 4). In the first day (19.03.2018) the AE energies were in general low (almost blue background), only one small green spot of bad correlation and a bit higher energies one can distinguish in the picture. It could be a warning of a small fault zone formation localized in the vicinity of the mining face. The next day of measurements (21.03.2018) we see a significant progression of faulting. The red and green spots of high $\mathrm{AE}$ energies and low values of correlation indicate that. And during the last day (23.03.2018) we observe the decay of AE energies without any anomaly to appear (blue background). 
a)

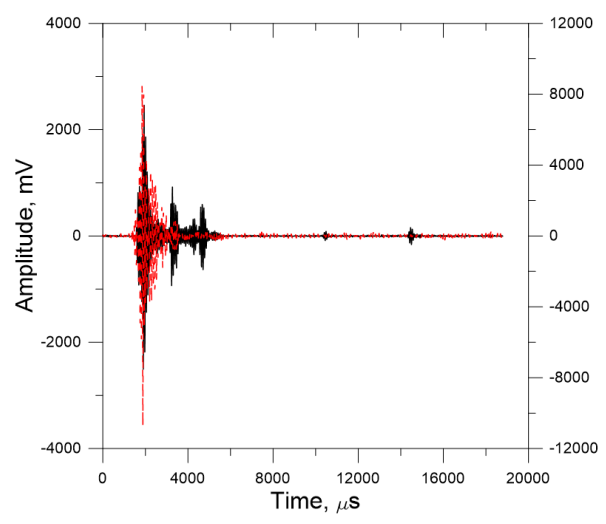

b)

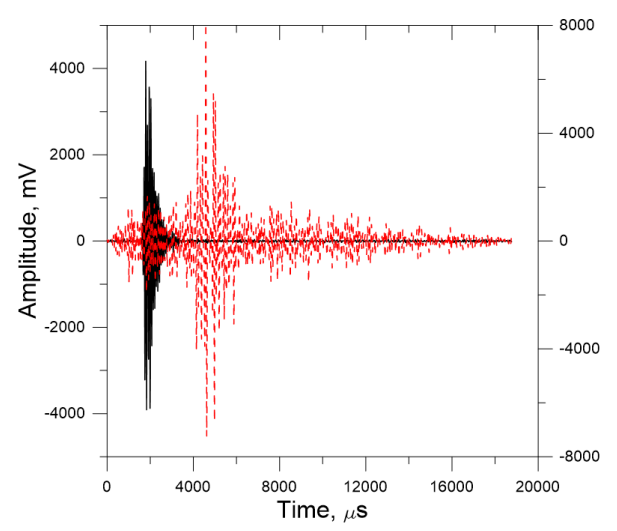

Fig.2. Examples of two pairs of AE waveforms: $a$ ) - perfect similarity; b) - bad similarity.

a)

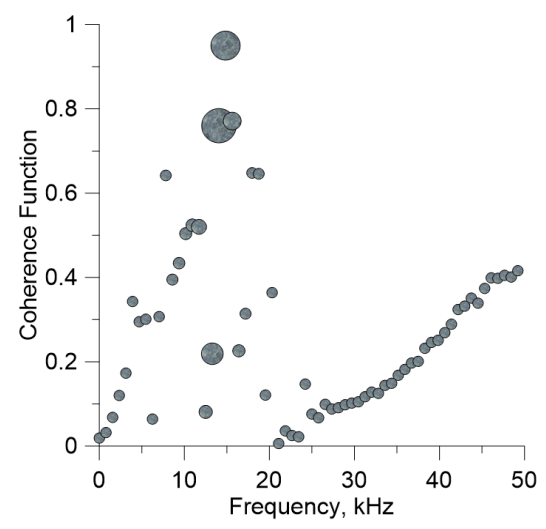

b)

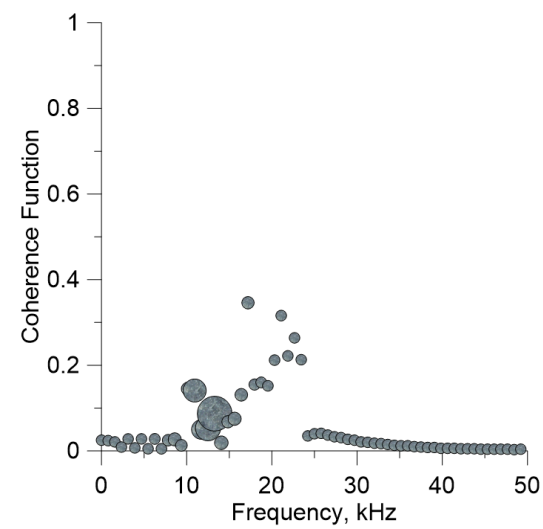

Fig.3. Cross-correlation analysis as an evaluation of AE waveform similarity: a) - perfect similarity; b) - bad similarity. 


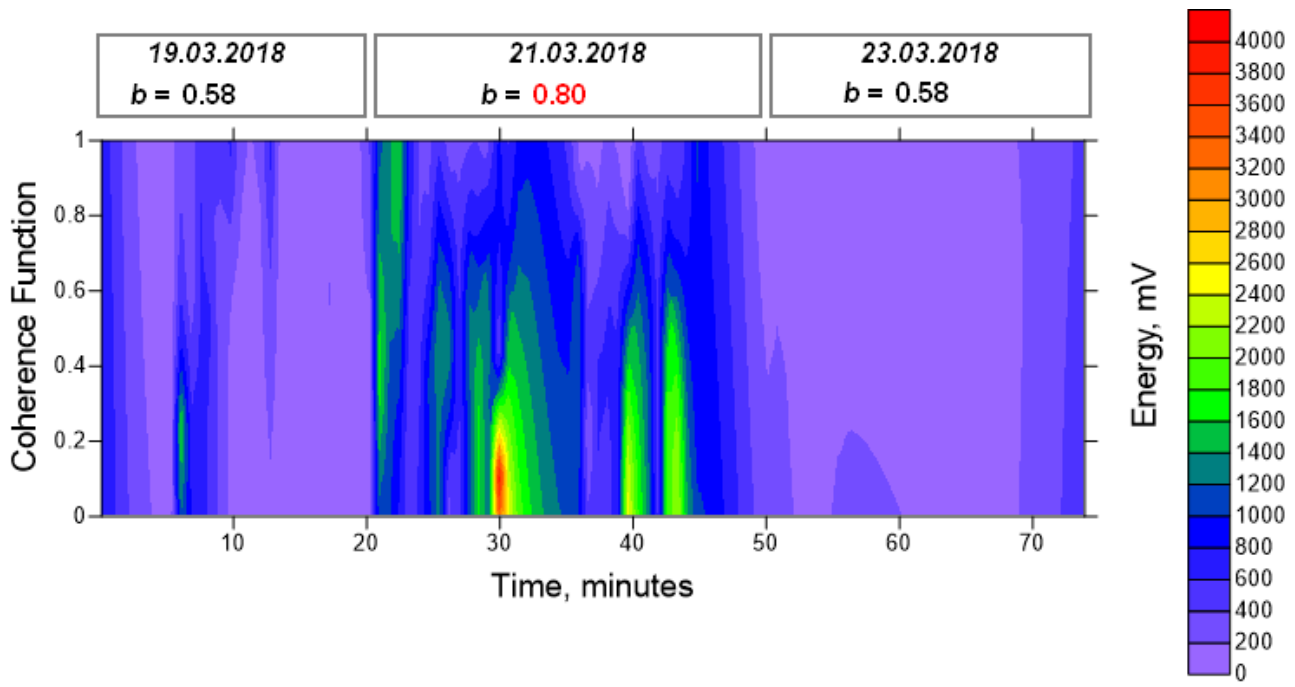

Fig.4. Time domain 3D display of AE monitoring in mine. Red spot is an indication of stress concentration process. The averaged $b$-values for each day are pointed out above.

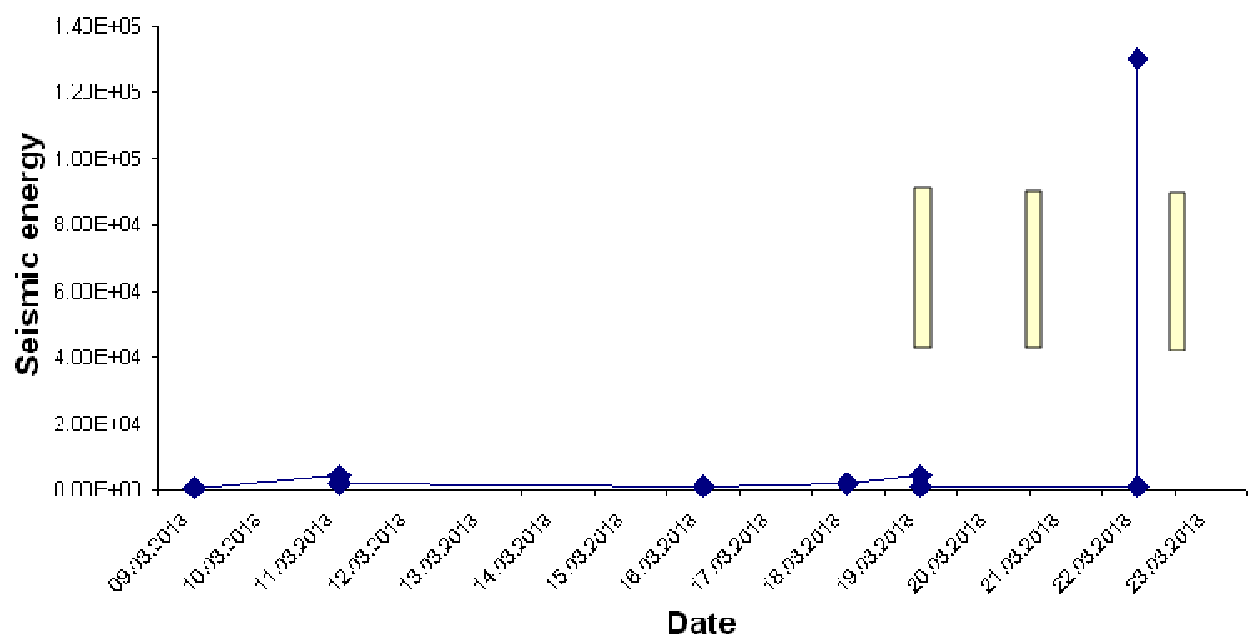

Fig.5. Seismic data obtained in the area where AE monitoring has been conducted. Blue diamonds depict the energy values, and yellow rectangles depict the days when $\mathrm{AE}$ monitoring has been carried out.

\section{Conclusions}

As a result we can make the following conclusions. In order to interpret AE waveform data we make an assumption that similar source mechanisms result in similar AE waveforms. Any change in similarity should produce different values of coherence function. Through the observation of coherence and energy trends we can control a transition to unstable phase of fracturing and take precautions during mining to reduce a risk of rock damage. 
In Figure 5 the seismic data for the area of AE monitoring is performed. On 22 of March two strong seismic events have been registered by the seismic acquisition system in the area of AE monitoring. Taking into account that our AE observations (21.03.2018) of intensification of fracturing process have preceded the strong seismic events, we can suggest that it was a process of strain accumulation and afterwards a process of relaxation associated with these strong seismic events.

\section{References}

1. Z. Reches, D.A. Lockner, J. Geophys. Res., 99, B9 (1994).

2. A. Zang, C. Wagner, S. Stanchits, C. Janssen, G. Dresen, J. Geophys. Res., 105, B10 (2000).

3. I.Yu. Rasskazov, D.S. Migunov, P.A. Anikin, A.V. Gladyr, A.A. Tereshkin, D.O. Zhelnin, J. Min. Sci., 51, 3 (2015).

4. A.O. Rozanov, Proc. Int. Geophys. Conf. \& Exhibition on Geophysics of the $21^{\text {st }}$ Century, Extended Abstracts, OS22 (2003).

5. A.O. Rozanov, Search and Discovery, \#40897 (2012).

6. K. Aki, P.G. Richards, Quantitative Seismology. Theory and methods (2 volumes), W.H. Freeman, San Francisco, 932 pp. (1980).

7. I.Yu. Rasskazov, S.V. Tsirel, A.O. Rozanov, A.A. Tereshkin, A.V. Gladyr, J. Min. Sci., 53, 2 (2017).

8. A.O. Rozanov, D.N. Petrov, A.M. Rozenbaum, A.A. Tereshkin, M.D. Ilinov, Geomechanics and Geodynamics of Rock Masses, pp. 669-672 (2018).

9. J.S. Bendat, A.G. Piersol, Random Data: Analysis and Measurement Procedures, WileyInterscience (1974).

10.J.S. Bendat, A.G. Piersol, Engineering Applications of Correlation and Spectral Analysis, John Wiley and Sons (1980). 